\title{
EDUCATION QUALITY DETECTION METHOD BASED ON THE PROBABILISTIC NEURAL NETWORK ALGORITHM
}

\author{
Changdong WU ${ }^{1}$, Hua JIANG ${ }^{2}$, Ping WANG ${ }^{1}$ \\ ${ }^{1}$ School of Electrical Engineering and Electronic Information, Xihua University, Chengdu, 610039, China \\ ${ }^{2}$ School of information science and technology, Southwest Jiaotong University, Chengdu, 610031, China \\ E-mail: ysx98042051@163.com, 25692877@qq.com, 99083094@qq.com
}

\begin{abstract}
The traditional education quality detection method is too single and unreasonable, which is not suitable to evaluate students' ability comprehensively. In this paper, the probabilistic neural network (PNN) algorithm is used to detect the education quality by considering the important impact between the various achievements of students. PNN algorithm originates from Bayesian decision rule, and it uses the non-linear Gaussian Parzen window as the probability density function. As PNN model has the virtues of strong nonlinear and anti-interfering ability, it is fit to detect the education quality by classifying the students' achievements. Besides, the influences of different evaluation models on classification accuracy and efficiency are also discussed in this paper. Furthermore, the effect of spread value on PNN model is also discussed. Finally, the actual data are used to detect the education quality. Experimental results show that the detection accuracy can reach $95 \%$, and the detection time is only $0.0156 \mathrm{~s}$ based on the proposed method. That is to say, the method is a very practical detection algorithm with high accuracy and efficiency. Moreover, it also provides a reference for how to further improve the teaching quality.
\end{abstract}

Keywords: detection; probabilistic neural network; classification; students' achievement; education quality

\section{INTRODUCTION}

With the rapid development of information technology, many universities use a professional education quality detection system to evaluate the students' ability. However, the existing detection system is inadequate in evaluating education quality effectively. In recent years, with the expansion of higher education and the rapid growth of the number of students, students' achievements produce a large number of data. So, it is necessary to use the comprehensive multiangle detection method to replace the conventional single detection method. At present, in view of the actual needs of the education quality detection system, it should use the advanced information technology to obtain effective evaluation from students' achievements, and thus find out the correlation between the students' achievements and abilities. That is to say, it is very important to evaluate the education quality comprehensively and objectively based on advanced detection method.

An important detection mean of education quality is to classify students' achievements. It's main purpose is build a proper classification model to analyze the effective information hidden in the students' achievements. As for now, there are many classification methods, such as moment invariants [1], random forest[2,3], support vector machine [4], principal component analysis[5], Markov random fields[6], particle swarm optimization[7], discrete wavelet transform[8], et al. Additionally, in [9], a novel framework of complex network classifier is proposed to tackle the problem of network classification, which shows that the proposed method performs well on large-scale networks. In [10], it uses the domain-adversarial learning method to classify the low-resource text, and it can obtain good results. In [11], a method to detect and classify ten short-circuit faults in the distribution networks is introduced, and it proves the effectiveness of the proposed method. In [12], A SAR image recognition algorithm is proposed by combining the convolution neural network and the extreme learning machine algorithm, and the accuracy of the image recognition is $100 \%$.

In addition to the above mentioned classification methods, the probabilistic neural network (PNN) model is a single-pass feed-forward neural network with the capability of providing the non-linear decision boundaries[13], which is suit for classification. PNN model is obtained by the Bayesian network and statistical principle. It originates from the radial basis function network. So, it learns more quickly than many other neural network models. As for now, it is widely used in varieties of applications such as detection [14-16], object tracking [17], diagnosis [18], classification [19], prediction [20], et al. In [14], a tool breakage detection system is proposed based on PNN model. Finally, it shows that the detection system can 
obtain the satisfactory results. In [17], a PNN model is used to detect camouflaged objects in video sequences, which makes the detection process faster and improves the detection accuracy. In [18], the paper introduces a method to heart activity diagnosis based on PNN model. In this method, the PNN model provides a powerful tool to classify the input data. In [21], PNN algorithm is introduced to solve the false detection problem of video advertising detection, and the experimental results show that the precision and recall are improved. In [22], PNN model is used as classifiers, which is more suitable for the prediction to olefin transstructure.

Furthermore, in order to improve the performance of PNN model, some combination methods based on PNN with other unique methods are introduced in many papers. In [23], optimal parameters of PNN are determined by means of the particle swarm optimization method. The results demonstrate the effectiveness of the proposed method. In [24], the introduced algorithm based on PNN and k-nearest neighbour methods is used to classify the extensive air shower data sets, the results validate that the method improves the classifying quality. In [25], a gas-path fault diagnosis method based on exergy loss and PNN model is introduced, and the results show that the diagnosis method can detect the fault and locate the malfunction component accurately. In [26], an improved PNN model is used to forecast the financial markets, which can get a higher accuracy and help stakeholders of stock market make accurate decisions. In [27], it proposes a load identification method based on PNN model. The results show that it can obtain the high accuracy in determining type of loads. In [28], a novel bearing fault diagnosis method based on PNN model with other methods is proposed for improving the accuracy of bearing fault recognition. The experimental results show that the fault recognition effect is good. In [29], a modified cuckoo search algorithm is proposed to globally optimize the parameter of PNN model, and the results show that the introduced method can improve the accuracy of fault diagnosis significantly. In [30], PNN model is considered for classification due to its accuracy rate with the statistical features, and the experimental results improve its efficiency. In addition, many other methods based on PNN model are used in practical applications, which obtain the encouraging results [31-33].

In this paper, the PNN model is used to classify the students' achievement. Experimental results indicate that the proposed method can improve the efficiency of classification so as to improve the teaching quality and promote the teaching reform.

The remainder of the paper is structured as follows: in section 2, PNN model and related concept are introduced. In section 3, it gives the experimental method, the discussions and analysis are presented based on experimental results. In section 4 , the conclusion is given.

\section{PROBABILISTIC NEURAL NETWORK ALGORITHM}

In the neural network technology, PNN model uses the exponential function to replace $S$-shape activation function. It is a feed-forward neural network, which is suit for data classification.

\subsection{The basic concept of PNN model}

The structure of PNN contains four layers such as input layer, pattern layer, summation layer and output layer. Its structure is shown in Fig. 1.

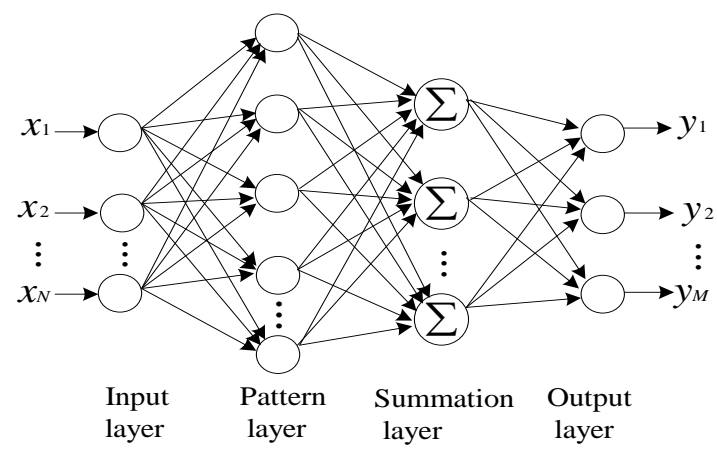

Fig. 1 Structure of PNN model

The effect of every layer is described as Fig. 2.

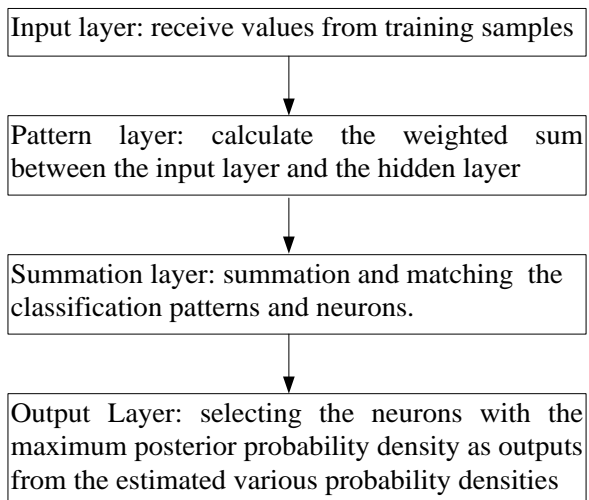

Fig. 2. The functions of each layer

The main advantages of PNN model include the following aspects:

(1) Simple structure and fast training speed, which is suit for real-time data processing.

(2) Each training sample determines a hidden layer neuron, and the weight value of neuron is directly taken from the input sample value.

(3) It can realize the nonlinear approximation arbitrarily, the classification result is insensitive to the form of basis function.

(4) No matter how complicated the classification process is, as long as there are enough training data, it can obtain the optimal solution under the Bayesian classification rule.

(5) It is no need to retrain when the new samples are added. 
(6) Use the linear learning algorithm to complete the intention of nonlinear learning algorithm.

In addition, it also has the virtues of high classification accuracy, good expansibility and good fault tolerance, et al.

Usually, the basic algorithm of PNN is shown in Fig. 3. Of course, it should be noted that PNN makes three assumptions for probability density function.

(1) The probability density function of each category is identical.

(2) The probability density function satisfies the Gaussian distribution.

(3) The variation matrix of Gaussian distribution probability density function is diagonal matrix, and the values of each diagonal element are equal.

PNN model has good characteristic based on the above-mentioned assumptions.

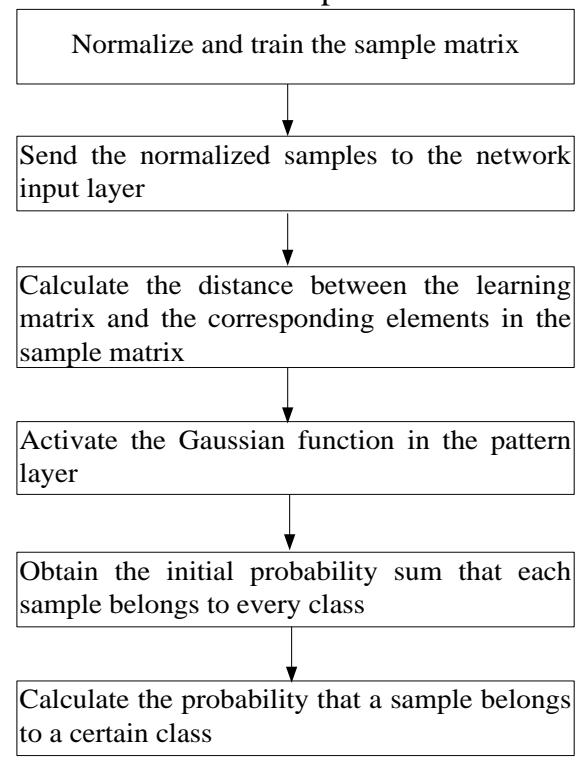

Fig.3. The basic algorithm of PNN

Concretely, the PNN algorithm can be described as follows:

(1) Use each sample as a vector, and build the initial training vectors from source data.

(2) The number of neurons in the input layer is equal to the dimension of the feature vectors. The input nodes are merely distribution nodes that provide the same input values to all pattern nodes, and the feature vectors are passed to each node in the pattern layer.

(3) The number of neurons within the pattern layer is equal to the number of input samples. When the input data are given, it will produce a vector. In this step, the activation function operator is expressed as:

$$
g\left(z_{j}\right)=\exp \left[\left(z_{j}-1\right) / \sigma^{2}\right]
$$

In Eq.(1), $z_{j}$ is the output value. In this step, every input sample value will be multiplied by a weight coefficient of the pattern layer.
The output value of each unit in pattern layer is expressed as:

$$
f_{\mathrm{A}}(X)=\sum \exp \left(-\frac{\left\|X-X_{i j}\right\|^{2}}{2 \sigma^{2}}\right)
$$

In Eq.(2), $X=\left[x_{1}, x_{2}, \ldots x_{n}\right]$ is the input sample, $X_{i j}$ is the weight matrix between the input layer and pattern layer, it means that $j$ training sample belongs to $i$ category, $i=1,2 \ldots M$, $j=1,2 \ldots N . M$ is the total number of samples, and $N$ is the number of category $i . \sigma$ is the smooth coefficient, which is the unique value that can be adjusted. Generally, $\sigma$ is set between 0 and 1.

The conditional probability of a sample attribution is expressed by probability density function.

$$
f_{A}(X)=\frac{1}{(2 \pi)^{n / 2} \sigma^{n}} \frac{1}{m} \sum \exp \left[-\frac{\left\|X-X_{i j}\right\|^{2}}{2 \sigma^{2}}\right] \text { (3) }
$$

In Eq. (3), $n$ is the dimension of sample space characteristic vectors, $m$ represents the number that samples belong to category $i$.

(4) As shown in Eq.(4), the summation layer connects all kinds of pattern layer units, and it selectively adds the probability that belongs to the same category in the same layer. In this layer, the number of neurons is equal to the number of samples.

$$
f_{A}=\sum_{i=1}^{m} g\left(z_{i}\right)
$$

(5) The output layer is a competitive neuron decision layer, where a competitive function selects the maximum probability which is the competitive output of the different probability density function, and each neuron corresponds to one category respectively. The number of neurons in the output layer is equal to the number of the training samples. Usually, the testing data are close to the sample data. Finally, only one neuron wins in the competing process. The neuron output with maximum probability is 1 , and the category is corresponding to the positive sample classification result, and the other neurons are the negative sample classification results.

\subsection{Classification method based on PNN model}

PNN model belongs to a supervised network classifier, it has the characteristics of fast training, good fault tolerance and accurate classification ability. The classification method based on PNN model can be described as follows:

Suppose there are two categories $\theta_{A}, \theta_{B}$. For every sample $X=\left[x_{1}, x_{2}, \ldots x_{n}\right]$, it belongs to $\theta_{A}$ or $\theta_{B}$.

It should satisfy the Bayesian rule: if $h_{A} l_{A} f_{A}(X)>h_{B} l_{B} f_{B}(X)$, then $X \in \theta_{A}$; 
if $h_{A} l_{A} f_{A}(X)<h_{B} l_{B} f_{B}(X)$, then $X \in \theta_{B}$.

Where, $h_{\mathrm{A}}$ and $f_{\mathrm{B}}$ are priori probability, and they meet the condition $h_{A}=N_{A} / N$, $h_{B}=N_{B} / N . N_{A}$ and $N_{B}$ are the number of training samples of $\theta_{A}$ and $\theta_{B}$ respectively. $N$ is the total number of training samples. $l_{A}$ and $l_{B}$ are the loss functions with the incorrect decision respectively. $f_{A}$ and $f_{B}$ are the probability density functions of the category $\theta_{A}$ and $\theta_{B}$ respectively.

PNN is an artificial neural network based on statistical principle. It absorbs the advantages of radial basis function and classical probability density function estimation principle. Compared with the traditional feed-forward neural network, it has more significant advantages in classification.

\section{EXPERIMENTS AND RESULTS}

Students' achievements classification has the significant role in detecting education quality. In order to evaluate the education quality objectively, fairly and reasonably, it is a very meaningful job to evaluate students according to their achievements accurately and properly. PNN model has the characteristics of fast classification speed, high accuracy and stable testing results. In this paper, we mainly establish the PNN model to find out the main factors that affect students' achievement. Besides, it classifies the students' achievements based on the diversified evaluation method so as to provide a guidance for the education reform.

Students' achievements classification includes the following several parts, which is shown in Fig.4.

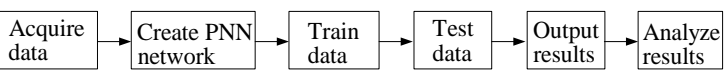

Fig. 4. The following chart of classification based on PNN model

In Fig. 4, students' achievements are acquired by dean's office, firstly. Then it creates PNN network based on above-introduced method. Afterwards, it trains and tests students' achievements. In this process, the training data and testing data are selected randomly to improve the accuracy of classification results. Finally, it obtains the experimental results, and the results are analyzed based on accuracy and efficiency, respectively. With the help of powerful characteristic of PNN, it can obtain satisfactory classification results.

Students' achievements mainly include usual behavior, home-work, mid-term examination and final examination, et al. In particular, usual behavior includes attendance $(20 \%)$, watching the MOOC video (15\%), interaction (10\%), discussion $(15 \%)$, reply $(15 \%)$, small experiments $(15 \%)$, brief essay $(10 \%)$. In fact, usual behavior can truly and effectively reflect students' learning state and learning ability comprehensively. Furthermore, because the diversified evaluation method can highlight students' comprehensive ability, a diversified classification guidance model based on the students' achievement is constructed. Table.1 shows the students' achievement distributions. These data come from the course "analog electronic technology", which is a provincial excellent online open course. Of course, it just shows part of the students' achievements.

Table 1. Students' achievements

\begin{tabular}{|c|c|c|c|c|c|}
\hline $\begin{array}{c}\text { Name } \\
\text { series }\end{array}$ & $\begin{array}{c}\text { Usual } \\
\text { behavior }\end{array}$ & $\begin{array}{c}\text { Home } \\
\text {-work }\end{array}$ & $\begin{array}{c}\text { Mid- } \\
\text { term }\end{array}$ & Final & Total \\
\hline 1 & 95 & 85 & 85 & 72 & 77 \\
\hline 2 & 85 & 85 & 83 & 73 & 76 \\
\hline 3 & 90 & 80 & 83 & 73 & 76 \\
\hline 4 & 60 & 60 & 80 & 67 & 67 \\
\hline 5 & 100 & 90 & 85 & 75 & 80 \\
\hline 6 & 94 & 95 & 79 & 65 & 72 \\
\hline 7 & 80 & 70 & 70 & 38 & 49 \\
\hline 8 & 95 & 85 & 75 & 66 & 72 \\
\hline 9 & 100 & 96 & 96 & 87 & 90 \\
\hline 10 & 95 & 79 & 82 & 66 & 72 \\
\hline 11 & 95 & 75 & 81 & 56 & 64 \\
\hline 12 & 60 & 60 & 68 & 69 & 67 \\
\hline 13 & 95 & 85 & 87 & 68 & 74 \\
\hline 14 & 100 & 94 & 92 & 84 & 87 \\
\hline
\end{tabular}

Divides students' achievements into five categories: excellent, good, medium, pass and failed. In fact, it is helpful to explore the students' potential ability and provide a beneficial reference value for education reform by evaluating students' achievements comprehensively.

As for PNN model, it contains many parameters. Different parameters have a great influence on its performance. Spread value is one of the important parameters, which has an important effect on testing accuracy. The effect of spread value on PNN is shown in Fig. 5.

In Fig. 5, it is obvious that the testing accuracy rate has a downward trend with the increasing of the spread value. Especially, when the spread value is set to 10 , the testing accuracy rate is only $63.33 \%$, which is not conducive to students' achievements classification. So, it is important to choose a reasonable spread value. In this paper, the spread value is set as 1 based on the above analysis.

Students' achievements are divided in five grades, which are corresponding to 15 models in PNN. The 15 models are shown in Table 2.

Different model will produce different testing accuracy. In order to demonstrate the ability of PNN in classifying students' achievement, the general regression neural network (GRNN) model is also used to classify students' achievement 
simultaneously, and the testing accuracy comparison diagram of 15 models is shown in Fig. 6.

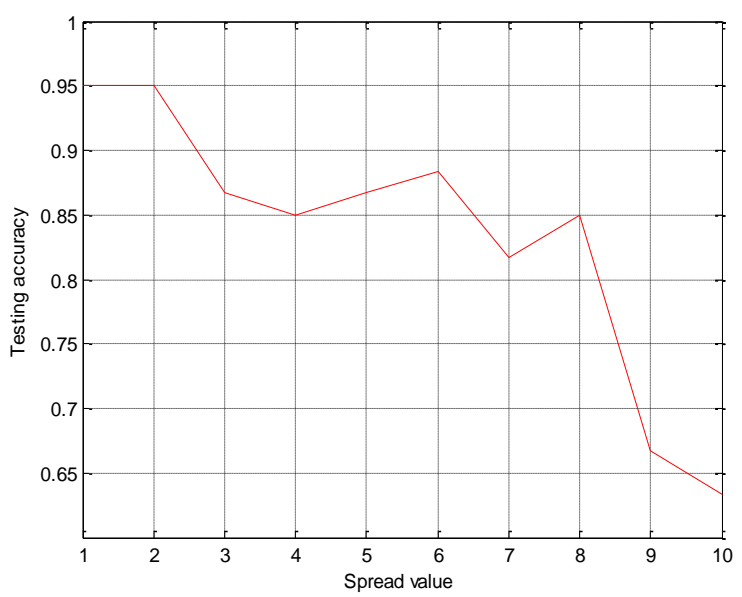

Fig. 5. The effect of spread value on PNN

Table 2. The corresponding relationship between model and input variables

\begin{tabular}{|c|l|l|l|l|l|}
\hline Grades & \multirow{2}{*}{ Excellent } & Good & Medium & Pas s & Failed \\
\hline 1 & $\checkmark$ & $\checkmark$ & $\checkmark$ & $\checkmark$ & $\checkmark$ \\
\hline 2 & & $\checkmark$ & $\checkmark$ & $\checkmark$ & $\checkmark$ \\
\hline 3 & & & $\checkmark$ & $\checkmark$ & $\checkmark$ \\
\hline 4 & & & & $\checkmark$ & $\checkmark$ \\
\hline 5 & & & & & $\checkmark$ \\
\hline 6 & & $\bullet$ & $\bullet$ & $\bullet$ & $\bullet$ \\
\hline 7 & & & $\bullet$ & $\bullet$ & $\bullet$ \\
\hline 8 & & & & $\bullet$ & $\bullet$ \\
\hline 9 & & & & & $\bullet$ \\
\hline 10 & & & $\mathbf{0}$ & $\mathbf{0}$ & $\mathbf{0}$ \\
\hline 11 & & & & $\mathbf{0}$ & $\mathbf{0}$ \\
\hline 12 & & & & & $\mathbf{0}$ \\
\hline 13 & & & & $\mathbf{0}$ & $\mathbf{0}$ \\
\hline 14 & & & & & $\mathbf{0}$ \\
\hline 15 & & & & & $\diamond$ \\
\hline
\end{tabular}

In Fig. 6, it can obtain higher testing accuracy using GRNN model than that of PNN model during the model 1 to model 12. However, in the model 14 and model 15, the testing accuracy based on PNN model are higher than that of GRNN model. Besides, it can only achieve the testing accuracy 93.33\% based GRNN model, while the highest testing accuracy can reach $95 \%$ based on PNN model.

Furthermore, according to the above testing results, we can make relevant suggestions on the students' learning situation. For example, as shown in table.1, No.2 and No.3 have the same total scores, but their usual behavior and home-work scores are different. So, we can put forward specific suggestions for the two students based on obtained results. In addition, we can put forward some opinions according to the rationality of students' classification, so that make students see their advantages and disadvantages and better plan their future study.

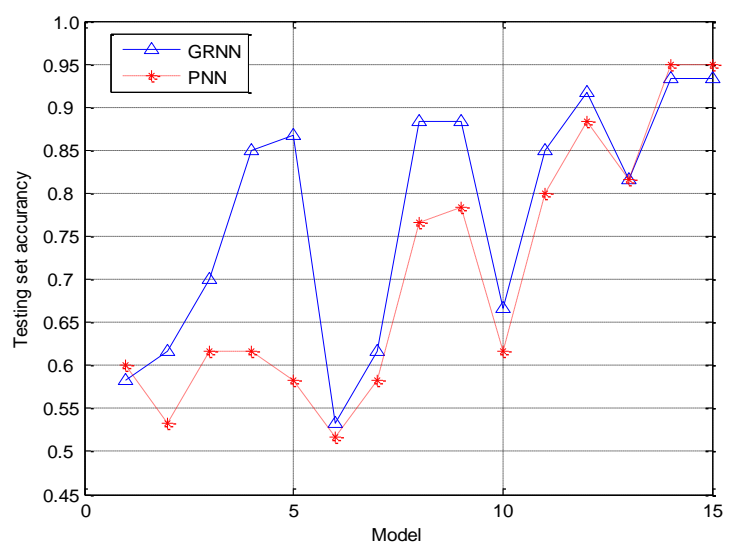

Fig. 6. Testing accuracy comparison diagram based on different model

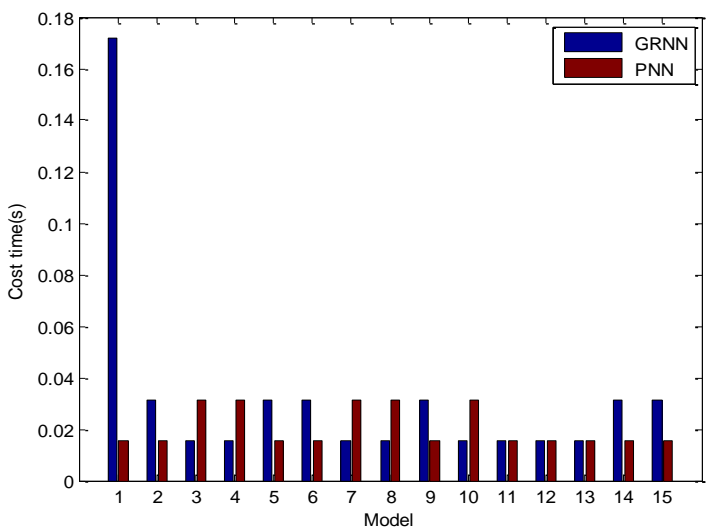

Fig. 7. Cost time comparison diagram based on GRNN and PNN models

As for a detection system, efficiency is another worth considering factor. Usually, using the different model will cost different time. As shown in Fig. 7, it will cost more time based on the GRNN model than PNN model. The longest time is $0.1716 \mathrm{~s}$ based on GRNN model, while the longest time is $0.0312 \mathrm{~s}$ and the shortest time is only $0.0156 \mathrm{~s}$ based on PNN model. It is obvious that the 14th and 15th models have significant advantages in classification efficiency.

According to the classification accuracy and efficiency, we use the 15th model of GRNN and PNN to classify the students' achievements, respectively. The classification result is shown in Fig. 8.

In Fig.8, there are 3 error categories during the 60 testing samples based on PNN model, and the 4 error categories happen in GRNN model. Besides, we classify the students' achievement using the PNN model, the cost time is only $0.0156 \mathrm{~s}$ and the classification accuracy is $95 \%$, this proves that it is an effective education quality detection method based on PNN model. 


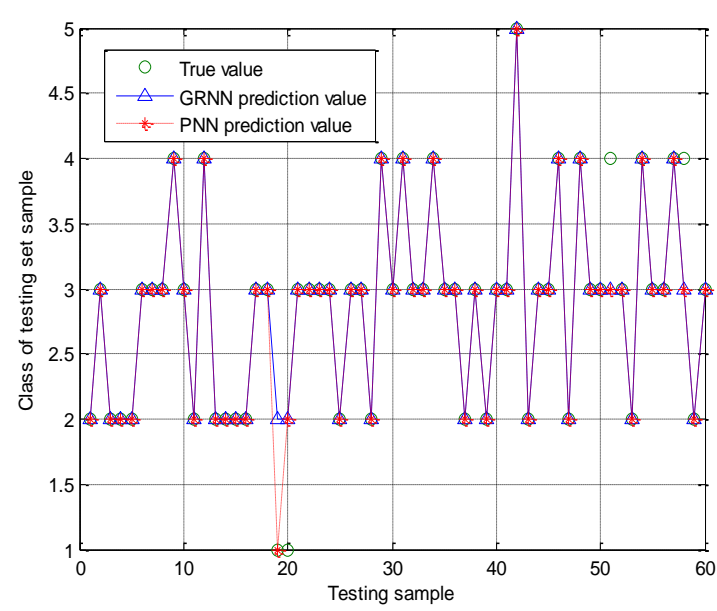

Fig. 8. The classification of testing set sample based on GRNN and PNN models

\section{CONCLUSION}

PNN model includes input layer, pattern layer, summation layer and output layer. It uses the Bayesian classifier and decision-making theory to build the system model. In this process, it uses the Gaussian non-linear function as activation function. It has the advantages of fast speed and high classification precision, so it is suitable for detecting education quality by classifying the students' achievements. In this paper, students' achievements and abilities are fully considered by using the PNN model to classify their achievements. Experimental results prove that the proposed method has high classification accuracy and efficiency. It is an effective and reasonable education quality detection method.

In the future, further research can be carried out from the following aspects:

(1) Increase the diversity of classes and the number of data samples.

(2) Increase the diversity of students' learning quality evaluation index.

(3) Apply advanced deep learning theory such as convolutional neural network and deep belief network and so on to analyze data samples, and use more methods to compare with the proposed method, so as to further improve the effectiveness of students' learning quality detection.

\section{ACKNOWLEDGEMENT}

This paper is supported by construction and research of online open course (MOOC) in colleges and universities of Sichuan Province (SCKC2019035); Research and reform project of undergraduate education and teaching in Southwest Jiaotong University (20201012-07); Ministry of education university-industry collaborative education program (201901103029, 201902020020). Higher education talent training quality and teaching reform project in Sichuan province from 2018 to 2020 (JG2018-571, JG2018-
578); Sichuan province "curriculum ideological and political" demonstration course "analog electronic technology" (2019-18); The third batch of provincial innovation and entrepreneurship education demonstration course ([2018]744-30); Sichuan Province excellent online open courses (Fundamentals of Analog Electronic Technology) (2019-64); Higher education talent training quality and teaching reform project of Xihua University (X2013JG0702).

\section{REFERENCES}

1. Mohamed Ben Haj Rhouma, Joviša Žunić, Mohammed Chachan Younis. Moment invariants for multicomponent shapes with applications to leaf classification. Computers and Electronics in Agriculture. 2017;142(A):326-337. https://doi.org/10.1016/j.compag.2017.08.029.

2. Fallatah Ahmad, Jones Simon, Mitchell David. Objectbased random forest classification for informal settlements identification in the Middle East: Jeddah a case study. International Journal of Remote Sensing. 2020;41(11): 4421-4445.

https://doi.org/10.1080/01431161.2020.1718237

3. Ko BC, Gim JW, Nam JY. Cell image classification based on ensemble features and random forest. Electronics Letters.2011;47 (11):638-U72. http://dx.doi.org/10.1049/el.2011.0831

4. Sarah N, Alyami,Sunday O. Olatunji.Application of Support Vector Machine for Arabic Sentiment Classification Using Twitter-Based Dataset.Journal of Information \& Knowledge Management. 2020;19 (01)2040018:1-13 https://doi.org/10.1142/S0219649220400183

5. Arivudainambi D, Varun Kumar KA, Sibi Chakkaravarthy $S$, et al. Malware traffic classification using principal component analysis and artificial neural network for extreme surveillance. Computer Communications. 2019;(147): 50-57. https://doi.org/10.1016/j.comcom.2019.08.003.

6. Hamid Ghanbari, Saeid Homayouni, Abdolreza Safari. et al. Gaussian mixture model and Markov random fields for hyperspectral image classification. European Journal of Remote Sensing. 2018; 51(1): 889-900. https://doi.org/10.1080/22797254.2018.1503565

7. Mirvaziri H, Mobarakeh ZS. Improvement of EEGbased motor imagery classification using ring topology-based particle swarm optimization. Biomedical Signal Processing and Control. 2017; (32): 69-75. https://doi.org/10.1016/j.bspc.2016.10.015

8. Anibou C, Saidi MN, Aboutajdine D. Classification of textured images based on discrete wavelet transform and information fusion. Journal of Information Processing Systems. 2015;11(3):421-437. https://doi.org/10.3745/JIPS.02.0028

9. Xin RY, Zhang J, Shao YT. Complex network classification with convolutional neural network. Tsinghua Science and technology. 2020;25(4): 447457.

https://dx.doi.org/10.26599/TST.2019.9010055

10. Griesshaber D, Vu NT, Maucher J. Low-resource text classification using domain-adversarial learning. Computer Speech and Language. 2020;62:101056. https://doi.org/10.1016/j.csl.2019.101056

11. Zhang Chao, Wang Jiandong, Huang Jian, et al. 
Detection and classification of short-circuit faults in distribution networks based on fortescue approach and softmax regression. International Journal of Electrical Power \& Energy System. 2020; 118-105812. https://doi.org/10.1016/j.ijepes.2019.105812

12. Wang P, Zhang XM, Hao Y. A method combining CNN and ELM for feature extraction and classification of SAR image. Journal of Sensors. 2019:6134610.

13. Naik, SM, Jagannath, RPK, Kuppili.V. Bat algorithmbased weighted Laplacian probabilistic neural network. Neural Computing \& Applications. 2020;32(4):

1157-1171. https://doi.org/10.1007/s00521-019-04475-4

14. Tsang BHP, Ma CC, Kuo CH. A PNN self-learning tool breakage detection system in end milling operations. Applied Soft Computing Journal. 2015;(37): https://doi.org/10.1016/j.asoc.2015.08.019

15. Yiming Huang, Shuaishuai Hou, Shufeng Xu,et al. EMD-PNN based welding defects detection using laser-induced plasma electrical signals. Journal of Manufacturing Processes.2019;(45):642-651. https://doi.org/10.1016/j.jmapro.2019.08.006.

16. Behnam M. Pourghassem H. Spectral correlation power-based seizure detection using statistical multiLevel dimensionality reduction and PSO-PNN optimization algorithm. IETE Journal of Research. 2017;63(5):736-753. https://doi.org/10.1080/03772063.2017.1308845

17. Mondal A, Ghosh S, Ghosh A. Partially camouflaged object tracking using modified probabilistic neural network and fuzzy energy based active contour. International Journal of Computer Vision. 2017;122(1)116-148. https://doi.org/10.1007/s11263-016-0959-5

18. Beritelli F, Capizzi G, Lo SG, et al. Automatic heart activity diagnosis based on Gram polynomials and probabilistic neural networks. Biomedical Engineering Letters. 2018;8(1):77- 85. https://doi.org/10.1007/s13534-017-0046-z

19. Kusy M, Kluska J. Assessment of prediction ability for reduced probabilistic neural network in data classification problems. Soft Computing. 2017;21(1): 199-212. https://doi.org/10.1007/s00500-016-2382-9

20. Xiaoyan Huang, Zhaoyong Guan, Li He, et al. A PNN prediction scheme for local tropical cyclone intensity over the South China Sea. Natural Hazards. 2015; 81(2):1-19. https://doi.org/10.1007/s11069-015-2132-9

21. Lan XL, Cui J, Zhang ST. Research on the video advertising detection based on PNN and text detection method. Journal of Information Hiding and Multimedia Signal Processing. 2017;8(5):1082-1091.

22. Yang AM, Han Y, Xing HW. Building SVM and PNN optimal classifiers based on GA-PLS algorithm and the application in infrared spectrum. International Journal of Advanced Media and Communication. 2016;6(2-4)198-210. https://dx.doi.org/10.1504/IJAMC.2016.080977

23. Porwik P, Doroz R, Orczyk T. Signatures verification based on PNN classifier optimised by PSO algorithm. Pattern Recognition. 2016;(60):998-1014. https://doi.org/10.1016/j.patcog.2016.06.032

24 Rastegarzadeh G, Nemati M. Primary mass discrimination of high energy cosmic rays using PNN and k-NN methods. Advances in Space Research. 2018;61(4) 1181 https://doi.org/10.1016/j.asr.2017.11.016

25 Cao Y, Lv X, Han G. et al. Research on gas-path faultdiagnosis method of marine gas turbine based on exergy loss and probabilistic neural network. Energies $\quad 2019 ;(12)$ : 4701. https://doi.org/10.3390/en12244701

26 Chandrasekara V, Tilakaratne C, Mammadov M. An improved probabilistic neural network model for directional prediction of a stock market index. Appl. Sci. 2019;(9): 5334 https://doi.org/10.3390/app9245334

27 Anggriawan DO, Amsyar A, Prasetyono E, et al. Load identification using harmonic based on probabilistic neural network. Emitter-International Journal of Engineering Technology. 2019;7(1): 71-82. https://doi.org/10.24003/emitter.v7i1.330

28 Zhang SJ, Zhao HM, Xu JJ, et al. A novel fault diagnosis method based on improved adaptive variational mode decomposition, energy entropy, and probabilistic neural network. Transaction of the Canadian Society for Mechanical Engineering. 2020;44(1): 121-132. https://doi.org/10.1139/tcsme2018-0195

29 Xie Zihao, Yang Xiaohui, Li Anyi. et al. Fault diagnosis in industrial chemical processes using optimal probabilistic neural network. Canadian Journal of Chemical Engineering. 2019;97(9):24532464.

https://doi.org/10.1002/cjce.23491

30 Mohanty Mihir Narayan, Palo Hemanta Kumar. Child emotion recognition using probabilistic neural network with effective features. Measurement. 2020;(152): 107369. https://doi.org/10.1016/j.measurement.2019.107369

31 Wang B, Ke HW, Ma XD, et al. Fault diagnosis method for engine control system based on probabilistic neural network and support vector machine. Applied Sciences-Basel. 2019;9(19): 4122. https://doi.org/10.3390/app9194122

32 Ying Xuxia, Tang Bibo, Zhou Canxin. Nursing scheme based on back propagation neural network and probabilistic neural network in chronic kidney disease. Journal of Medical Image and Health informatics. 2020;10(2): 416-421. https://doi.org/10.1166/jmihi.2020.2879

33 Fan Hongguang, Pei Jihong, Zhao Yang. An optimized probabilistic neural network with unit hyperspherical crown mapping and adaptive kernel coverage. Neurocomputing. 2020;(373): 24-34. https://doi.org/10.1016/j.neucom.2019.09.029

Received 2020-04-10

Accepted 2020-09-02

Available online 2020-11-17

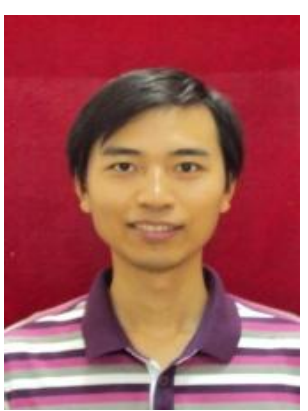

Changdong WU, a researcher in the School of Electrical Engineering and Electronic Information, Xihua University. His research area is signal and information processing. Especially, he is interested in image processing, pattern recognition, non-destructive testing method based on the image processing technique. 


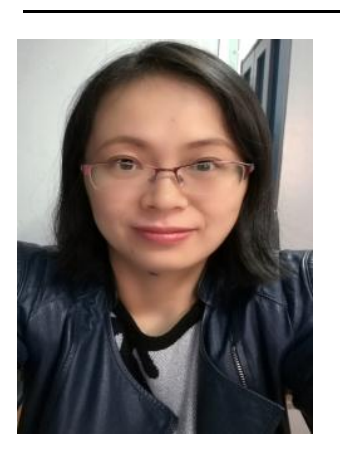

Hua JIANG, a researcher in the school of information science and technology, Southwest Jiaotong

University. Her research area is signal and information processing.

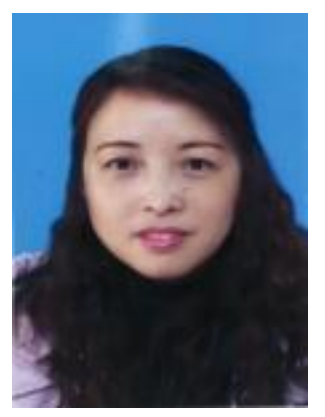

Ping WANG, a researcher in

the School of Electrical Engineering and Electronic Information, Xihua University. Her research area is signal detection and conversion. 